\title{
GANGRENE OF THE EXTREMITIES IN TWO AFRICAN INFANTS
}

\author{
BY \\ LORNA G. MACDOUGALL \\ From the King George VI Hospital, Nairobi
}

(RECEIVED FOR PUBLICATION NOVEMBER 18, 1954)

Gangrene of the extremities in infancy and childhood is a rare condition which has aroused the interest of previous clinicians because of its diverse and obscure aetiology. The two cases recorded here presented a very similar clinical picture in that the gangrene occurred in association with an acute gastro-intestinal infection.

\section{Case Reports}

Case 1. A male Mkamba child aged 5 months was admitted to King George VI Hospital, Nairobi, on March 24, 1954, with a history of diarrhoea and vomiting of three days' duration.

On admission the child appeared well nourished but was dehydrated and toxic: the temperature was $97^{\circ} \mathrm{F}$. $\left(36 \cdot 1^{\circ}\right.$ C.), pulse $120 / \mathrm{min}$., respirations $28 / \mathrm{min}$. Examination of the ears, throat, chest, and cardiovascular systems revealed no abnormalities. The mouth was dry and the abdomen lax and sunken. An intraperitoneal infusion of $0.45 \%$ saline with $2.5 \%$ glucose $(270 \mathrm{ml}$.) was given, gastric lavage performed, and feeding with Hartmann's solution started. Sulphaguanidine, $0.5 \mathrm{~g}$. six-hourly, was given by mouth and retained. Six loose green stools were passed during the next 12 hours, but a stool culture yielded no pathogenic organisms. No malarial parasites were detected in the blood slide.

By March 26 the hydration had improved, diarrhoea had decreased, but vomiting persisted. The child held himself stiffly and resented any interference. Although no fresh clinical signs were detected and the child remained afebrile, a course of penicillin, 20,000 units daily, was introduced. Lumbar puncture was performed and yielded a clear colourless fluid under normal pressure: cells, 10 lymphocytes per ml.; protein, $160 \mathrm{mg}$. \%; chlorides, $970 \mathrm{mg} .{ }^{\%}$; sugar, $156 \mathrm{mg} . \%$. No organisms were detected. In view of these findings a further intraperitoneal drip was given and the child's condition improved. Apart from occasionally vomiting sulphaguanidine, he retained feeds, and the diarrhoea subsided. On March 29 the temperature rose suddenly to $103^{\circ} \mathrm{F}$. $\left(39 \cdot 4^{\circ} \mathrm{C}\right.$.), and gangrene of both hands and the left ear was noticed. There was a clear line of demarcation at the junction of the ear and the scalp, and across the palms of both hands. Vomiting began again and the child died the same evening.
Post-mortem examination revealed a terminal bronchopneumonia involving both lungs. The peritoneal cavity contained no free fluid and showed no evidence of inflammation. There was moderate congestion of the intestinal tract, but no mucosal ulceration. No abnormality was detected in the heart or great vessels, and no macroscopic thromboses or emboli in the peripheral vessels. Unfortunately in this case no sections of blood vessels were taken for microscopic examination.

Case 2. A female Kikuyu child aged 3 months was admitted to King George VI Hospital, Nairobi, on May 23, 1954, with a history of diarnoea for the past 36 hours. The stools were loose and had been noticed to contain blood and mucus. On admission she appeared toxic and moderately dehydrated, with loss of tissue turgor. Her temperature was $98^{\circ} \mathrm{F}$. $\left(36 \cdot 7^{\circ} \mathrm{C}\right.$.), pulse $100 / \mathrm{min}$., respirations $24 / \mathrm{min}$. No abnormalities were detected in chest, cardiovascular system, throat, or ears. The abdomen was soft and peristalsis active. A stool culture on admission yielded Shigella flexneri. An intraperitoneal drip of $0 \cdot 45 \%$ saline with $2 \cdot 5 \%$ glucose $(270 \mathrm{ml}$.) was given, and sulphaguanidine, $0.5 \mathrm{~g}$. sixhourly, was taken by mouth. Hourly feeds of glucose saline were taken well and retained, but as hydration was unsatisfactory a further intraperitoneal drip was given on May 24. By May 27 the hydration had improved, but the child remained toxic and the diarrhoea persisted. On the morning of May 28 she developed gangrene of both feet and the right hand with a clear line of demarcation at ankles and wrist. By this time her general condition had improved considerably; she was feeding well, and the diarrhoea was less. As she showed every sign of immediate survival, and it was considered that the loss of both feet and the right hand would be an almost insuperable disability to an African girl in later life, it was decided to try heparin as a means of re-establishing the circulation. A regime of heparin, 1,000 units, together with penicillin, 100,000 units, sixhourly was therefore instituted. On May 29 it was noticed that the gangrene had spread above the right wrist, but the general condition gave no cause for concern until midday when the child suddenly collapsed and died.

At necropsy the body was that of a well-nourished child showing gangrene of both feet, the right hand, and 
lower third of the forearm. Examination of the abdomen revealed acute congestion of the bowel. The mucous membrane of both large and small intestines was red and velvety, and there were numerous enlarged mesenteric nodes. The mucous membrane of the stomach was injected, and the stomach contained some altered blood. The peritoneal cavity contained no free fluid. The liver was pale and fatty, but the other abdominal organs showed no macroscopic abnormality. The lungs were healthy and aerated. There was no evidence of a congenital cardiac abnormality or thrombosis of the great vessels. Examination of the arterial system from the abdominal aorta to the dorsalis pedes showed no macroscopic evidence of thrombosis. A similar negative result was obtained on dissection of the right brachial and radial arteries. There was, however, thrombosis of the veins accompanying the peripheral arteries. The upper limit of the thrombosis coincided with the line of demarcation of gangrene in the feet, but extended about one inch $(2.5 \mathrm{~cm}$.) above the level of demarcation in the forearm. Serial sections of the peripheral veins and arteries showed recent thrombus formation in the veins, but no evidence of arterial thrombosis, arteritis, or periarteritis.

\section{Discussion}

A survey of the literature describing gangrene in the early years of life provides an interesting study in clinical pathology. The incidence of the disease seems to be relatively higher among newborn babies than in the later months of infancy, but there is no common causative factor in either group.

Heller and Alvari (1941) report one case and have reviewed 39 other cases among newborn babies. Six were thought to have resulted from infection, five from pressure, seven from a possible Raynaud's disease or other neurological disorder, four from thrombosis, and one each from a doubtful syphilis and diabetes. In the remainder the cause was obscure. The suggested aetiology in their own case, which recovered, was that there has been an 'intimal agglutination' in the artery, which eventually became recanalized due to the pulsating column of blood acting proximal to the occlusion.

Other cases in infants have followed thrombosis and pressure (Brown and Smith, 1951), embolism or thrombosis from congenital heart lesions (Craig and Muns, 1931; Hansen, 1949), extension of the normal process of umbilical closure to involve the common iliac and hypogastric arteries (Garrod, Batten, and Thursfield, 1949), local infection (Alders, 1954), proved syphilis (Foster and Taylor, 1949), the inadvertent injection of nikethamide into the umbilical artery (Mills, 1949), and systemic infection in which microscopic inflammatory changes were observed in the arteries after death (Dohan, 1934).

Among the cases recorded in later infancy and childhood, systemic infection seems to play a greater part. In the series of von Khantz (quoted by Heller and Alvari, 1941) 39 cases were directly attributable to such infections as diphtheria, typhoid fever, scarlet fever, syphilis, pneumonia, measles, and enteritis. Similar findings have been recorded by Martin and Shore (1928), who reported four cases following diphtheria, pericarditis, indolent ulceration of the foot, and chronic tuberculosis respectively. In these cases a local endarteritis was demonstrated microscopically and occurred above the line of demarcation of the gangrene. Hoyne and Smollar (1941), who reported a case of gangrene associated with scarlet fever, also demonstrated arterial changes in the nature of a periarteritis. Lewis (quoted by Gelfand, 1947) described a bilateral symmetrical gangrene of the digits in childhood which was frequently associated with malnutrition, chronic ill health, or acute infection. The onset was sudden, the gangrene dry, and it was thought to be due to damage to the intima of the digital arteries causing thrombosis, although the main arteries to the limbs were unobstructed.

Gelfand (1947 and 1949) reported eight cases of symmetrical gangrene in adult Africans, thought to be due to a nutritional disturbance of the vessel wall leading to a vascular occlusion and gangrene. He also recorded one case in an African child in which thrombophlebitis was the causative factor. A recent case report published by Said (1954) of gangrene in a Malayan child presented a very similar clinical picture to the present cases in that the spontaneous gangrene of the feet occurred during the course of a gastro-intestinal infection.

In assessing the possible cause in the present cases, several factors were taken into consideration: (1) the nutritional state of the infants; (2) the state of hydration; (3) the fact that the initial regime of treatment involved the use of an intraperitoneal infusion; and (4) the fact that both infants were suffering from a gastro-intestinal infection.

(1) Although malnutrition, both frank and subclinical, is often a complicating factor in African paediatrics, it is rarely seen in infants under 6 months unless the breast-milk supply is inadequate or the child is suffering from some debilitating disease. Both these infants were well nourished, the mothers had adequate milk, and there was no previous history of illness. Gelfand's theory of nutritional vascular changes was therefore considered inapplicable.

(2) Spontaneous venous thrombosis is known to occur in severely dehydrated infants, particularly in the sluggish venous sinuses in the brain. It was thought at the time, and without any previous 
experience of infantile gangrene, that a peripheral thrombosis due to dehydration had been the operative factor in Case 1, but the fact that the child's hydration had improved considerably 48 hours before the onset of the gangrene made the conclusion unsatisfactory. It is noted by Foster and Taylor (1949) that such peripheral circulatory disorders, when they do occur in infants, are often of a temporary nature and that the changes are usually reversible.

(3) Intraperitoneal infusions have been used in this hospital for approximately two years with very satisfactory results, and in no case has death or any serious complication been directly attributable to this form of therapy (Carter, 1953). It has been stressed, however, that it is essential to have the fluid warmed to body heat before transfusion in order to prevent the occurrence of shock in an already cold and dehydrated child. It was thought possible, therefore, that the introduction of cold fluid into the peritoneal cavity might have produced spasm in the large vessels of the posterior abdominal wall, thus precipitating thrombosis in an already sluggish circulation. This factor was thought worthy of consideration because in both cases there was reason to doubt whether the technique of transfusion had been faultless: in Case 1 the first drip was undertaken hurriedly owing to a delay in obtaining the saline, and in Case 2 the first drip was given as an emergency measure in another ward, unaccustomed to the technique, before the child was transferred to the children's ward. But, as both infants had had further intraperitoneal therapy under adequate supervision and did not develop gangrene until five days thereafter, it is doubtful if this could have been the sole factor in precipitating the condition.

(4) In previous cases of gangrene associated with acute infections, the condition is said to have been due to thrombosis following a toxic arteritis, and this has been proved histologically in several instances. It is thought that circulating toxins or micro-organisms cause damage to the endothelial lining of the vessel walls with subsequent thrombosis. Normally the arterial stream would be too rapid to permit prolonged contact of micro-organisms with the vessel walls sufficient to produce damage, but in the capillary network and vasa vasorum the stream is much slower, and it is presumed that the process of thrombosis starts in these minute vessels and eventually spreads to involve the arterioles (Martin and Shore, 1928).

\section{Conclusion}

From the foregoing résumé of the literature and in the absence of any other demonstrable cause, it is suggested that gangrene in the cases here reported was due to a capillary thrombosis in which both spasm and infection played a part.

\section{Summary}

Two fatal cases of peripheral gangrene occurring in African infants suffering from gastro-enteritis are described. The literature relating to peripheral gangrene in childhood is briefly reviewed.

\section{REFERENCES}

Alders, N. (1954). Archives of Disease in Childhood, 29, 160.

Brown, R. J. K. and Smith, S. R. N. (1951). Ibid., 26, 574.

Carter, F. S. (1953). E. Afr. med. J., 30, 499.

Craig, J. D. and Muns, W. E. (1931). Amer. J. Dis. Child., 41, 126. Dohan, F. C. (1934). J. Pediat., 5, 756.

Foster, W. P. and Taylor, R. G. G. (1949). Archives of Disease in Childhood, 24, 152 .

Garrod, A. E., Batten, F. E. and Thursfield, H. (1949). Diseases of Children, 4th ed., ed. D. Paterson and A. Moncrieff, vol. 2, p. 689.

Gelfand, M. (1947). Brit. med. J., 1, 847.

Gelfand, M. (1947). Brit. med. J., 1, 847.

Hansen, J. D. L. (1949). Archives of Disease in Childhood, 24, 149

Heller, G. and Alvari, G. (1941). Amer. J. Dis. Child., 62, 133.

Hoyne, A. L. and Smollar, L. (1941). J. Pediat., 18, 242.

Martin, W. and Shore, B. R. (1928). Am. Surg., 88, 725.

Mills, W. G. (1949). Brit. med. J., 2, 464.

Said, M. (1954). Med. J. Malaya, 8, 263. 